\title{
ON NONLOCAL MODELING IN CONTINUUM MECHANICS
}

\begin{abstract}
The objective of the paper is to provide an overview of nonlocal formulations for models of elastic solids. The author presents the physical foundations for nonlocal theories of continuum mechanics, followed by various analytical and numerical techniques. The characteristics and range of practical applications for the presented approaches are discussed. The results of numerical simulations for the selected case studies are provided to demonstrate the properties of the described methods. The paper is illustrated with outcomes from peridynamic analyses. Fatigue and axial stretching were simulated to show the capabilities of the developed numerical tools.
\end{abstract}

Keywords: solid mechanics, long-range force interactions, nonlocal theory of elasticity, nonlocal modeling

\section{MODELOWANIE NIELOKALNE W MECHANICE KONTINUUM}

$W$ artykule dokonano przegladu nielokalnych sformułowań dla mechaniki bryly odksztatcalnej. Autor przedstawia podstawy fizyczne nielokalnych teorii mechaniki kontinuum oraz dokonuje przegladu technik analitycznych $i$ numerycznych stosowanych $w$ modelach matematycznych. W pracy przedyskutowano charakterystyki oraz zakres praktycznych zastosowań wspomnianych technik modelowania. Ocena własności nielokalnych modeli została przeprowadzana na podstawie wyników symulacji numerycznych dla wybranych typów analiz z zastosowaniem perydynamiki. Przedstawiono wyniki symulacji zmęczeniowych oraz jednoosiowego rozciagania, uzyskane przy użyciu opracowanych narzędzi analiz symulacyjnych.

Stowa kluczowe: mechanika ciała stałego, nielokalne siły interakcji, nielokalne sformułowanie teorii sprężystości, modelowanie nielokalne

\section{INTRODUCTION}

Foundations for the classical mechanics of continuum solid media refer to the theory of linear elasticity, which is governed by locally formulated relationships between stresses and strains. Although consequently applied for decades, this theory suffers from several incapabilities with regards to the completeness of its description of the physical behavior of deformable solids observed at various length scales. To name a couple of such-related physical phenomena, shear bands while stretching and the dispersion of waves were unexpectedly discovered during the experiments, which cannot be derived from the classical formulations of solid mechanics (Eringen, Edelen 1972; Kaliski et al. 1992; Di Paola et al. 2010). Hence, alternative theories emerged to aim at avoiding the inconsistency between the theory and experiments, which made successful attempts to use the nonlocal formulation of elasticity (Kröner 1967; Kunin 1967; Eringen 1972).

Nonlocal components, which are considered in the governing equations, allow for the unique properties of an analytical or numerical model (Eringen 1972). First, a length scale can be explicitly introduced to ensure a more-physical behavior of the model. This property of the model applies to the phenomena, which manifest their presence at different geometric scales. Due to the involved nonlocality, it is therefore feasible to address the contribution of microstructure in the resultant macroscale continuous model (Kunin 1983; Chen et al. 2004; Di Paola et al. 2010). Second, the phenomenon of elastic wave propagation in deformable solids can be handled more accurately via the nonlocal approach without the need for a further increase of mesh density (Eringen 1972). Having introduced nonlocal interactions into a modeled structure, one can conveniently form the shape of the dispersion curves and surfaces (Martowicz et al. 2015a). Effectively, the model is considered as a periodic structure being investigated within the first Brillouin zone (Martowicz et al. 2014a). The theory of generalized continua also reflects the nonlocal properties of solids modeled as granular media at the micro-scale and nano-scale (Ostoja-Starzewski 2013). A similar approach was also adapted for upscaling techniques (Seleson et al. 2009).

The nonlocal modeling technique was extensively investigated in different research areas and for various physical domains. As reported, the nonlocal approach may efficiently reduce numerical dispersion, which is crucial for the proper determination of physical dispersion (Ghrist 2000; Tam, Webb 2011; Yang et al. 2012). Moreover, nonlocality opened up new perspectives for modeling evolving damages (Rodriguez-Ferran et al. 2004; Gunzburger, Lehoucq 2010), studying vibro-acoustic

* AGH University of Science and Technology, Faculty of Mechanical Engineering and Robotics, Department of Robotics and Mechatronics, Krakow, Poland; e-mail: adam.martowicz@agh.edu.pl 
wave interaction (Martowicz et al. 2014c), and allowing us to regularize boundary value problems (Bazant, Jirasek 2002). Introducing nonlocal interactions increases the quality of a mesh by allowing for the more-spontaneous growth of a modeled crack (Hu et al. 2012). The nonlocal theory of elasticity was successfully applied to model graphene, via both the nonlocal finite element method (FEM) (Arash et al. 2012) and peridynamics (Martowicz et al. 2015b), piezoelectricity (Eringen 1984; Zhang et al. 2014), and shape memory alloys (Badnava et al. 2014), taking into account superelasticity (Duval et al. 2010). Nonlocal formulations for thermoelasticity are addressed in (Eringen 1974; Balta, Suhubi 1977), including some recent works (Chang, Wang 2015). The paper is organized as follows. After the introduction (which is given in the present section), Section 2 provides the physical foundations for nonlocal modeling, followed by both analytical and numerical methods (which are briefly described in Section 3). The results of numerical studies are presented and discussed in Section 4. Final conclusions are drawn in Section 5 .

\section{PHYSICAL FOUNDATIONS}

The classical theory of elasticity does not make any references to the granular nature of physical matter. Hence, it prevents real long-range interactions, which are present at the nano-scale and micro-scale. A common and convenient approach within the classical approach is to homogenize the properties of the material over a finite domain and provide the resultant macro- and possibly meso-scale model, which is considered valid when subjected to long wave propagation (Eringen, Edelen 1972). The description of such a model involves local stress-strain relationships. However, the experimental works regarding dispersion curves for phonons propagating in metallic structures clearly state the necessity of introducing nonlocal formalisms into the governing equations (Eringen 1972).

If more-accurate (i.e., physical) descriptions are demanded in a model, and the phenomena observed at the nano- and micro-scales are expected to be accounted for, an alternative nonlocal theory should be adapted and used, as it reflects potential-based reactions (which are nonlocal by nature). This requirement seems crucial, especially for the studies on crack growth, fatigue, and acoustic emission. Ultimately, a modeled solid acts like a periodic granular media at the micro- and nano-scales (Martowicz et al. 2015b). The size effect may be effectively handled by a nonlocal model as well, and various shapes of the dispersion relationships may be recreated when the model is subjected to the propagation of elastic waves of different lengths. The size of a neighboring area for the interacting pieces of a deformable body is tuned to assure the proper representation of the phenomena present at different length scales. This approach allows the microstructure's mechanisms to be included into the resultant behavior of a meso- or macro-scale model. The nonlocal modeling is accomplished by nonlocal formulations introduced into constitutive models, allowing us to handle the real deviations observed in the experiments due to the above-mentioned inner microstructure (Di Paola et al. 2010).

Nonlocality is a critical issue also in the case of modeling shape memory alloys. Only the proper selection of a neighboring area for long-range interactions during the martensitic phase transformation enables the modeling strain instabilities observed during the experiments (Duval et al. 2010). The size effect again plays an important role and should be considered in the modeling. In the following section, examples of nonlocal modeling approaches are briefly presented to show how the long-range interactions are considered.

\section{ANALYTICAL AND NUMERICAL MODELING}

There have been various approaches proposed to account for the nonlocality in the models of a solid body. Depending on the formulation of a given kernel function (which is used to introduce constitutive relationships), the following types of nonlocal governing equations - based on spatial integro-differential-based expressions - are considered in contrast to the classical local Cauchy problem:

- integro-differential-based function composition (Duruk et al. 2010):

$$
\frac{\partial^{2} u(x, t)}{\partial t^{2}}=\frac{\partial^{2}}{\partial x^{2}} \int_{\Re} \beta(x-\hat{x}) g(u(\hat{x}, t)) d \hat{x}
$$

where $\beta$ is an integrable kernel function and $g$ denotes a nonlinear function of displacement $u$ for the considered one-dimensional case. $x$ and $\hat{x}$ are, respectively, the actual central and neighboring localizations for the interacting portions of a solid;

- integro-differential expression with a nonlocal formulation of the Cauchy problem (Eringen 1992):

$\rho(x) \frac{\partial^{2} \mathbf{u}(\mathbf{x}, t)}{\partial t^{2}}=\nabla \cdot \sigma(\mathbf{u}(\mathbf{x}, t))+\mathbf{b}(\mathbf{x}, t)$

with a nonlocal formulation for stress tensor $\sigma ; \rho$ and $\mathbf{b}$ denote mass density and external body force, respectively; 
- integro-differential expression proposed for a generic volume element in a one-dimensional case (Zingales 2011):

$$
\begin{aligned}
\rho(x) A(x) \frac{\partial^{2} u(x, t)}{\partial t^{2}}-\frac{\partial}{\partial x}\left[E_{n l}(x) A(x) \frac{\partial u(x, t)}{\partial x}\right]+ \\
+\int_{a}^{b} g(x, \xi) \eta(x, \xi, t) d \xi=A(x) f(x, t)
\end{aligned}
$$

where $A$ and $E_{n l}$ are the cross-sectional area and Young's modulus, respectively; $g$ is the kernel, which depends on a distance-decaying function; function $\eta$ is the relative displacement determined at coordinates $x$ and $\xi, f$ denotes the external body force field;

- nonlocal integral formulation for peridynamics (Silling 2000):

$$
\rho \frac{\partial^{2} \mathbf{u}(\mathbf{x}, t)}{\partial t^{2}}=\int_{H} \mathbf{f}(\mathbf{u}(\hat{\mathbf{x}}, t)-\mathbf{u}(\mathbf{x}, t), \hat{\mathbf{x}}-\mathbf{x}) d V_{\hat{\mathbf{x}}}+\mathbf{b}(\mathbf{x}, t)
$$

with pairwise force $\mathbf{f}$, which acts within finite domain $H$ and depends on both the relative position $\hat{\mathbf{x}}-\mathbf{x}$ in the reference coordinate system and the relative displacement $\mathbf{u}(\hat{\mathbf{x}}, t)-\mathbf{u}(\mathbf{x}, t)$.

All of the above-mentioned expressions introduce nonlocal components that allow for an extended range of interacting forces within the modeled body. Hence, this approach makes a direct reference to the potential-based interactions for granular media as experimentally identified at the nano- and micro-scales. The similarity of these expressions to those applied for the nano- and micro-structures enables us to introduce specific, experimentally proven behavior that cannot be derived from the classical local Cauchy problem description for solids.

It should be noted that the peridynamics does not introduce spatial partial derivatives (in contrast to the remaining nonlocal approaches), which helps us avoid numerical inconveniences at the geometric discontinuities. Hence, spontaneous crack growth may be modeled more physically. It is also worth mentioning that higher-order spatial partial derivatives are a common approach used to introduce a length scale into a governing equation, which is necessary for modeling phenomena at different geometric scales (Seleson et al. 2009). Capturing nonlocality via domain decomposition and variational calculus techniques is shown in (Di Paola et al. 2010; Aksoylu, Parks 2011).

To complement the above-listed nonlocal analytical approaches, various discrete and numerical methods should also be considered. The best-known techniques are molecular dynamics (Seleson et al. 2009) and cellular automata with extended interaction regions; i.e., a secondary von Neumann neighbor, as reported in (Leamy, Springer 2011). The micropolar and Cosserat theories (Chen et al. 2004), extensions of FEM (Polizzotto 2001; Arash et al. 2012), and finite difference method (FDM) (Sguazzero, Kindelan 1990; Fornberg 1998) were also proposed to address nonlocal descriptions for solid mechanics.

In the case of numerical simulations, there are two important aspects that should be taken into account in the context of accuracy and computational time. First, when a reliable numerical model is built, a convergence analysis is required. For nonlocal models, however, both the average distance between the degrees of freedom (DOF) and horizon for nonlocal interactions are required to be determined simultaneously (Bobaru et al. 2009). Second, nonlocality opens the perspective of parallel processing since, by its nature, a number of contributing forces or their equivalent quantities must be independently found for the entire interaction region for each simulation time step. Data processing using a graphics processing unit (GPU) naturally suits the numerical algorithms developed for nonlocal methods.

\section{NUMERICAL CASE STUDIES}

Various applications of numerical algorithms based on nonlocal methods have been proposed over the past few decades. One of the most-recent (i.e. peridynamics) is especially worth mentioning, as it gives us the opportunity to accurately model wave-based phenomena, including the regions of geometric discontinuities. The applications of peridynamics (which were used to solve problems regarding acoustic emission), clapping phenomenon, higher order harmonic waves generations, and propagation and reflection of Lamb's waves can be found in (Martowicz et al. 2014b). A vibro-acoustic wave interaction solution using the nonlocal approach is presented in (Martowicz et al. 2014c). The results of simulations for strain wave propagation in composite materials are reported in $(\mathrm{Hu}$ et al. 2012). A recent overview on the practical applications of peridynamics can be found in (Madenci, Oterkus 2014). Below, the outcomes for a two-dimensional (2-D) peridynamic model of an aluminum plate undergoing uniaxial stretching and fatigue are shown.

Numerical simulations were used to analyze the behavior of the models made of a homogeneous, isotropic material. The governing equation used to describe a 2-D numerical model is derived from Equation (4) and takes 
the following form for the $i$-th particle (Martowicz et al. 2014c):

$$
\left\{\begin{array}{l}
\rho \frac{\partial^{2} u_{i}(t)}{\partial t^{2}}=\sum_{j \in H_{i}}\left(\left(\xi_{X_{i, j}}+u_{j}(t)-u_{i}(t)\right) F_{i, j}(t) c A_{i, j} T\right)+b_{X_{i, j}} \\
\rho \frac{\partial^{2} v_{i}(t)}{\partial t^{2}}=\sum_{j \in H_{i}}\left(\left(\xi_{Y_{i, j}}+v_{j}(t)-v_{i}(t)\right) F_{i, j}(t) c A_{i, j} T\right)+b_{Y_{i, j}}
\end{array}\right.
$$

where $u_{i}(t)$ and $v_{i}(t)$ are the in-plane displacements of the particles along the $0 X$ and $0 Y$ axes. Horizon $H_{i}$ determines the population of the $j$-th neighboring particles interacting with the $i$-th actual central one. $\xi_{X_{i, j}}, \xi_{Y_{i, j}}, b_{X_{i, j}}$, and $b_{Y_{i, j}}$ stand for two pairs of relative particle positions and the components of the external body force. $A_{i, j}$ determines the area of the $j$-th particle covered by horizon $H_{i}$. The thickness of the model is defined with parameter $T$. Contributing factor function $F_{i, j}(t)$ takes the following form:

$$
\begin{aligned}
& F_{i, j}(t)=\frac{1}{\sqrt{\xi_{X_{i, j}}^{2}+\xi_{Y_{i, j}}^{2}}}- \\
&-\frac{1}{\sqrt{\left(\xi_{X_{i, j}}+u_{j}(t)-u_{i}(t)\right)^{2}+\left(\xi_{Y_{i, j}}+v_{j}(t)-v_{i}(t)\right)^{2}}}
\end{aligned}
$$

The elastic properties of the plate are defined with micromodulus function $c$ :

$$
c=\frac{6 E}{\pi \delta^{3}(1-v) T}
$$

which is found based on Young's modulus E, Poisson's ratio $v$, and the radius of circular horizon $\delta$. Having introduced Equation (5) to govern the behavior of an aluminum plate, a peridynamic model was prepared (with dimensions of $4 \times 10.125 \mathrm{~mm}$ ), as shown in Figure 1 . Its thickness equals $1 \mathrm{~mm}$.
The model satisfies a free-free condition. The forces, which are used to initiate an axial deformation of the plate, are spread within the two areas, each consisting of five columns of contributing vectors, and localized at the vertical edges of the model to prevent nonphysical point concentrations in the external body excitation. A single 0.37 -mm-long vertical crack is introduced in the model. The material properties are as follows: $E=70 \mathrm{GPa}$, $v=0.3, \rho=2100 \mathrm{~kg} / \mathrm{m}^{3}$. The model considers both the breaking of links between the particles when the ultimate stress (40 MPa) is exceeded as well as the contact mechanism, which allows for the temporal recovery of the force reactions when the edges of the developing crack approach each other. This model's formulation leads to a bilinear stiffness, which is observed for fatigue analysis. The temporal and spatial discretizations used in the model are determined with parameters $\Delta t=20 \mathrm{~ns}$ and $\Delta x=0.125 \mathrm{~mm}$, respectively. The radius of the horizon corresponds to a distance of $\delta=4 \Delta x=0.5 \mathrm{~mm}$ (Hu et al. 2012). An implicit time integration scheme was applied to determine the consecutive particle displacement. A criterion based on the maximum error regarding the displacements (i.e., $0.1 \mathrm{~nm}$ ) was used to terminate the iteration procedure for each time step.

Two case studies were investigated to track the path of the developing crack; these are shown in Figure 2.

1) Axial stretching with the time rise factor for force amplitude $\Delta F_{0} / \Delta t=1 \mathrm{MN} / \mathrm{s}$. The final value of the amplitude equals $F_{0}=100 \mathrm{~N}$.

2) Fatigue with force amplitude $F_{0}=35 \mathrm{~N}$ and frequency $f=200 \mathrm{kHz}$. The corresponding force amplitude's time rise factor equals $\Delta F_{0} / \Delta t=28 \mathrm{MN} / \mathrm{s}$. The simulation assumes that the amplitude of the sinusoidal force gradually rises within period $\tau=4 \mathrm{~ms}$ after the simulation is initiated. Hence, this determines that value $F_{0}$ is effectively achieved and then kept until the simulation terminates (within one-fourth of the period of a sinusoidal force excitation).

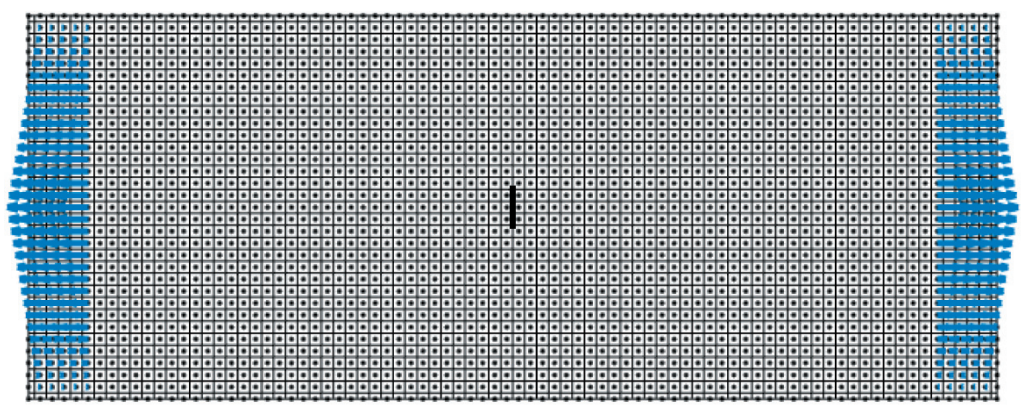

Fig. 1. Peridynamic model of aluminum plate used for stretching and fatigue analysis. Model considers centrally localized vertical crack and two areas of exciting forces 
a)

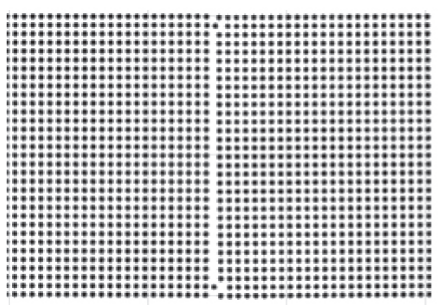

b)

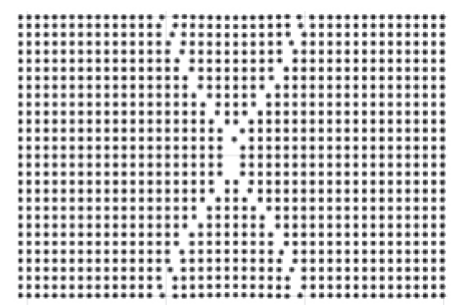

Fig. 2. Evolution of crack's path shown for central part of model, undergoing two types of force excitations: a) straight path observed for axial stretching, captured at $100 \mu \mathrm{s}$; b) skew paths for fatigue observed at $7 \mu \mathrm{s}$.

For better visualization, displacements shown are scaled by 50 with respect to actual ones

The exemplary results of the peridynamic simulations that are shown in the paper reflect the mechanisms of the growing and branching cracks (crack bifurcation) identified for various excitation regimes (Hu et al. 2012). Depending on both the increase ratio and nominal value of amplitude of the force acting on a deformable body, different physical behaviors of an emerging crack may be found. This observation refers to the experimental results, as in the case of high-strain deformations for composites described in (Haque, Ali 2005; Meyers, Chawla 2009). Since the definition of a peridynamic model allows for integral and potential-based formulations (i.e., a more-physical description of the interactions in an elastic body at the micro- and nano-scales), it is expected to model the crack's growth reliably and the phenomenon of its bifurcation employ more-realistic scenarios. The present work shows the straight and skew paths for a growing crack in a model with ongoing slow axial stretching as well as the fatigue where the different rise factors for the amplitudes of the forces are applied.

\section{CONCLUDING REMARKS}

Over recent decades, a rapid growth of interests regarding nonlocal methods for continuum mechanics has been observed. Among other applications of nonlocal formulations for solid mechanics, the modeling of crack and crack-wave simulations are of particular interest, since the above-mentioned methods characterize unique properties. As shown in the work, the capability of convenient modeling geometric discontinuities results in more physical paths (i.e., spontaneous paths) of emerging cracks not explicitly governed by any direction in a structured mesh. The potential-based problem description reflects the physical force dependencies between the pieces of a deformable solid body in a more-realistic way, which is demanded in reliable simulations of the crack's growth (e.g., in fatigue analyses).
The paper presents examples of the results of axial stretching and fatigue obtained with a peridynamic model. Different types of force excitations, varying in the time increase ratio and the nominal value of amplitude, lead to different paths of the crack's growth. Either the straight or skewed propagation's directions are successfully identified in the work to refer to already-published works, including the conducted experiments. From a practical point of view, it should be highlighted that the nonlocal methods are by their nature capable of applying the multithreading technique. The numerical algorithms that have been developed for the nonlocal methods for continuum mechanics can be effectively implemented in a GPU. The calculations required for each time step can be easily dispatched for each DOF undergoing many parallel nonlocal interactions. New values of nodal quantities can be found solving independent tasks simultaneously.

\section{References}

Aksoylu B., Parks M.L., 2011, Variational theory and domain decomposition for nonlocal problems. Applied Mathematics and Computation 217, 6498-6515.

Arash B., Wang Q., Liew K.M., 2012, Wave propagation in graphene sheets with nonlocal elastic theory via finite element formulation. Computer Methods Applied Mechanics and Engineering 223-224, 1-9.

Badnava H., Kadkhodaei M., Mashayekhi M., 2014, A non-local implicit gradient-enhanced model for unstable behaviors of pseudoelastic shape memory alloys in tensile loading. International Journal of Solids and Structures 51(23-24), 4015-4025.

Balta F., Suhubi E.S., 1977, Theory of nonlocal generalised thermoelasticity. International Journal of Engineering Science 15(9-10), 579-588.

Bazant Z.P., Jirasek M., 2002, Nonlocal integral formulations of plasticity and damage: survey of progress. Journal of Engineering Mechanics 128(11), 1119-1149.

Bobaru F., Yang M., Alves L.F., Silling S.A., Askari E., Xu J., 2009, Convergence, adaptive refinement, and scaling in $1 D$ peridynamics. International Journal for Numerical Methods in Engineering 77, 852-877.

Chang D.M., Wang B.L., 2015, Surface thermal shock cracking of a semi-infinite medium: a nonlocal analysis. Acta Mechanica 226, 4139-4147. 
Chen Y., Lee J.D., Eskandarian A., 2004, Atomistic viewpoint of the applicability of microcontinuum theories. International Journal of Solids and Structures 41, 2085-2097.

Di Paola M., Pirrotta A., Zingales M., 2010, Mechanically-based approach to non-local elasticity: Variational principles. International Journal of Solids and Structures 47, 539-548.

Duruk N., Erbay H.A., Erkip A., 2010, Global existence and blow-up for a class of nonlocal nonlinear Cauchy problems arising in elasticity. Nonlinearity 23, 107-118.

Duval A., Haboussi M., Zineb T.B., 2010, Modeling of SMA superelastic behavior with nonlocal approach. Physics Procedia 10, 33-38.

Eringen A.C., 1972, Linear theory of nonlocal elasticity and dispersion plane waves. International Journal of Engineering Science 10, 425-435.

Eringen A.C., 1974, Theory of nonlocal thermoelasticity. International Journal of Engineering Science 12(12), 1063-1077.

Eringen A.C., 1984, Theory of nonlocal piezoelectricity. Journal of Mathematical Physics 25(3), 717-727.

Eringen A.C., 1992, Vistas of nonlocal continuum physics. International Journal of Engineering Science 30(10), 1551- 1565.

Eringen A.C., Edelen D.G.B., 1972, On nonlocal elasticity. International Journal of Engineering Science 10, 233-248.

Fornberg B., 1998, Calculation of weights in finite difference formulas. SIAM Review 40(3), 685-691.

Ghrist M.L., 2000, High-order finite difference methods for wave equations. $\mathrm{PhD}$ thesis, University of Colorado.

Gunzburger M., Lehoucq R.B., 2010, A nonlocal vector calculus with application to nonlocal boundary value problems. Multiscale Modeling and Simulation 8(5), 1581-1598.

Haque A., Ali M., 2005, High strain rate responses and failure analysis in polymer matrix composites - an experimental and finite element study. Journal of Composite Materials 39, 423-450.

Hu W., Ha Y.D., Bobaru F., 2012, Peridynamic model for dynamic fracture in unidirectional fiber-reinforced composites. Computer Methods in Applied Mechanics and Engineering 217-220, 247-261.

Kaliski S., Rymarz C., Sobczyk K., Włodarczyk E., 1992, Vibrations and waves, Part B: Waves. Studies in Applied Mechanics 30B, Elsevier, Amsterdam - London - New York - Tokyo, PWN Polish Scientific Publishers, Warsaw.

Kröner E., 1967, Elasticity theory of materials with long range cohesive forces. International Journal of Solids and Structures 3(5), 731-742.

Kunin I.A., 1967, Inhomogeneous elastic medium with non-local interactions. Journal of Applied Mechanics and Technical Physics $8(3), 60-66$.

Kunin I.A., 1983, Elastic media with microstructure II: Three-dimensional models. Springer-Verlag, Berlin - Heidelberg.

Leamy M.J., Springer A.C., 2011, Parallel implementation of triangular cellular automata for computing two-dimensional elastodynamic response on arbitrary domains. Vibration Problems ICOVP 2011. The $10^{\text {th }}$ International Conference on Vibration Problems, Náprstek J., Horácek J., Okrouhlík M., Marvalová B., Verhulst F., Sawicki J.T. (eds.), Springer Proceedings in Physics $139,731-736$.

Madenci E., Oterkus E., 2014, Peridynamic theory and its applications. Springer, New York.
Martowicz A., Ruzzene M., Staszewski W.J., Rimoli J.J., Uhl T., 2014a, A nonlocal finite difference scheme for simulation of wave propagation in $2 D$ models with reduced numerical dispersion. Proceedings of SPIE 9064, Health Monitoring of Structural and Biological Systems 2014, Article ID 90640F, doi: 10.1117/12.2045252.

Martowicz A., Ruzzene M., Staszewski W.J., Uhl T., 2014b, Non-local modeling and simulation of wave propagation and crack growth. AIP Conference Proceedings 1581, 513, AIP Publishing.

Martowicz A., Staszewski W.J., Ruzzene M., Uhl T., 2014c, Vibro-acoustic wave interaction in cracked plate modeled with peridynamics. A Proceedings of the WCCM XI, ECCM V, ECFD VI, Onate E., Oliver X., Huerta (eds.), International Center for Numerical Methods in Engineering, Barcelona, Spain, 4021-4027.

Martowicz A., Ruzzene M., Staszewski W.J., Rimoli J.J., Uhl T., 2015a, Out-of-plane elastic waves in $2 D$ models of solids: A case study for a nonlocal discretization scheme with reduced numerical dispersion. Mathematical Problems in Engineering 2015, Article ID 584081

Martowicz A., Staszewski W.J., Ruzzene M., Uhl T., 2015b, Peridynamics as an analysis tool for wave propagation in graphene nanoribbons. Proceedings of SPIE, Volume 9435: Sensors and Smart Structures Technologies for Civil, Mechanical, and Aerospace Systems, Lynch J.P. (ed.), San Diego, USA, Article ID 94350I, doi: $10.1117 / 12.2084312$.

Meyers M., Chawla K., 2009, Mechanical behaviour of materials. Cambridge University Press, Cambridge.

Ostoja-Starzewski M., 2013, From random fields to classical or generalized continuum models. Procedia IUTAM 6, 31-34.

Polizzotto C., 2001, Nonlocal elasticity and related variational principles. International Journal of Solids and Structures 38, 7359-7380.

Rodriguez-Ferran A., Morata I., Huerta A., 2004, Efficient and reliable nonlocal damage models. Computer Methods in Applied Mechanics and Engineering 193, 3431-3455.

Seleson P., Parks M.L., Gunzburger M., Lehoucq R.B., 2009, Peridynamics as an upscaling of molecular dynamics. Multiscale Modeling and Simulation 8(1), 204-227.

Sguazzero P., Kindelan M., 1990, Dispersion-bounded numerical integration of the elastodynamic equations with cost-effective staggered schemes. Computer Methods in Applied Mechanics and Engineering 80(1-3), 165-172.

Silling S., 2000, Reformulation of elasticity theory for discontinuities and long-range forces. Journal of Mechanics Physics of Solids 48, 175-209.

Tam C.K.W., Webb J.C., 2011, Dispersion-relation-preserving finite difference schemes for computational acoustics. Journal of Computations Physics 107(2), 262-281.

Yang D., Tong P., Deng X., 2012, A central difference method with low numerical dispersion for solving the scalar wave equation. Geophysical Prospecting 60, 885-905.

Zhang L.L., Liu J.X., Fang X.Q., Nie G.Q., 2014, Effects of surface piezoelectricity and nonlocal scale on wave propagation in piezoelectric nanoplates. European Journal of Mechanics - A/Solids $46,22-29$.

Zingales M., 2011, Wave propagation in 1D elastic solids in presence of long-range. Journal of Sound and Vibration 330, 3973-3989. 